CRYSTALLOGRAPHIC COMMUNICATIONS

ISSN 2056-9890

Received 12 March 2017

Accepted 16 March 2017

Edited by $\mathrm{H}$. Stoeckli-Evans, University of Neuchâtel, Switzerland

Keywords: crystal structure; L-tyrosine; L-amino acid $\mathrm{N}$-carboxy anhydride; O-benzyl-L-tyrosine NCA; solid-state polymerization; hydrogen bonding; $\mathrm{C}-\mathrm{H} \cdots \pi$ interactions.

CCDC reference: 1538344

Supporting information: this article has supporting information at journals.iucr.org/e

\section{Crystal structure of $\mathrm{O}$-benzyl-L-tyrosine- $\mathrm{N}$-carboxy anhydride}

\author{
Aya Inada and Hitoshi Kanazawa* \\ Faculty of Symbiotic Systems Science, Fukushima University, 1 Kanayagawa, Fukushima, 960-1296, Japan. \\ *Correspondence e-mail: kana@sss.fukushima-u.ac.jp
}

In the title compound, $\mathrm{C}_{17} \mathrm{H}_{15} \mathrm{NO}_{4}$ \{alternative name: $(S)$-4-[4-(benzyloxy)benzyl]oxazolidine-2,5-dione\}, the oxazolidine ring is planer, with an r.m.s. deviation of $0.039 \AA$. The benzyloxy and benzyl rings are almost coplanar, making a dihedral angle of $0.078(10)^{\circ}$, and are inclined to the oxazolidine ring by $59.16(11)$ and $58.42(11)^{\circ}$, respectively. In the crystal, molecules are linked by $\mathrm{N}-\mathrm{H} \cdots \mathrm{O}$ and $\mathrm{C}-\mathrm{H} \cdots \mathrm{O}$ hydrogen bonds, forming ribbons propagating along [010]. The ribbons are linked by $\mathrm{C}-\mathrm{H} \cdots \pi$ interactions, forming a threedimensional supramolecular structure. The oxazolidine rings of adjacent ribbons are arranged into a layer parallel to the $a b$ plane. This arrangement is favourable for the polymerization of the compound in the solid state.

\section{Chemical context}

$\mathrm{N}$-Carboxy anhydrides (NCAs) of amino acids are extensively used as monomers for the preparation of high molecular weight polypeptides (Kricheldorf, 2006). Amino acid NCAs are easily soluble but the resulting polypeptides are not soluble in general organic solvents. Only a few amino acid ester NCAs such as $\gamma$-benzyl-L-glutamate NCA (BLG NCA) and $\beta$-benzyl-L-aspartate NCA (BLA NCA) are polymerized in solutions, because the resulting polypeptides are soluble in them. On the other hand, we found that every amino acid NCA crystal is polymerized in the solid state in hexane by the initiation of amines. We studied the polymerization of BLA NCA (Kanazawa \& Sato, 1996) and $\beta$-benzyl-DL-aspartate NCA (BDLA NCA) initiated by a primary amine in the solution and solid states, and we determined the crystal structure of BLA NCA (Kanazawa \& Magoshi, 2003) and BDLA NCA (Kanazawa \& Inada, 2017) to consider their high reactivity in the solid state. In addition, we prepared single crystals of the title compound, $O$-benzyl-L-tyrosine (OBLT NCA) in hexane-ethyl acetate mixture. The polymerization of OBLT NCA is initiated by butyl amine initiator in dioxane or acetonitrile solutions. However, the polymerization rate was extremely slow, because the resultant polymer has a poor solubility in these solvents. On the other hand, the polymerization of OBLT NCA initiated by butyl amine was very reactive in the solid state in hexane. High molecular weight poly(OBLT) was obtained only in the solid-state polymerization. High molecular weight poly(OBLT) is valuable, because poly(L-tyrosine) is obtained by the hydration of benzyl groups of the polymer. Therefore, it is important to determine the crystal structure to consider the difference in the reactivity in solution and in the solid state. 


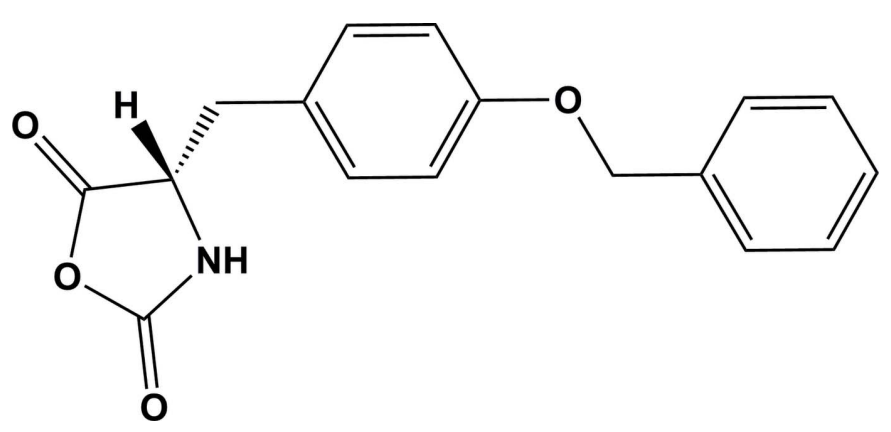

\section{Structural commentary}

The molecular structure of the title compound is shown in Fig. 1. The oxazolidine ring $(\mathrm{O} 2 / \mathrm{N} 1 / \mathrm{C} 1-\mathrm{C} 3)$ is planar with an r.m.s. deviation of $0.039 \AA$, and a maximum deviation of 0.033 (2) $\AA$ for atom C3. The side chain has an extended conformation with the $\mathrm{C} 3-\mathrm{C} 4-\mathrm{C} 5-\mathrm{C} 6$ and $\mathrm{C} 7-\mathrm{C} 8-\mathrm{O} 4-$ C11 torsion angles being $98.8(2)$ and $179.01(18)^{\circ}$, respectively. Hence, the benzyloxy (C12-C17) and benzyl (C5-C10) rings are almost coplanar, making a dihedral angle of $0.078(10)^{\circ}$, and are inclined to the oxazolidine ring by $59.16(11)$ and $58.42(11)^{\circ}$, respectively.

\section{Supramolecular features}

In the crystal, molecules are linked via $\mathrm{N} 1-\mathrm{H} 1 \cdots \mathrm{O} 3^{\mathrm{i}}$ and $\mathrm{C}-$ $\mathrm{H} \cdots \mathrm{O} 3^{\mathrm{ii}}$ hydrogen bonds, forming ribbons propagating along the $b$-axis direction (Table 1 and Fig. 2). The ribbons are linked by $\mathrm{C}-\mathrm{H} \cdots \pi$ interactions, forming a three-dimensional supramolecular structure (Table 1 and Fig. 3). The fivemembered oxazolidine rings are packed in a layer and the $-\mathrm{CH}_{2} \mathrm{C}_{6} \mathrm{H}_{4} \mathrm{OCH}_{2} \mathrm{C}_{6} \mathrm{H}_{5}$ side chains are packed in another layer; the two different layers stack alternately. This sandwich structure is one of the important requirements for high reactivity in the solid state, because the five-membered rings can react with each other in the layer.

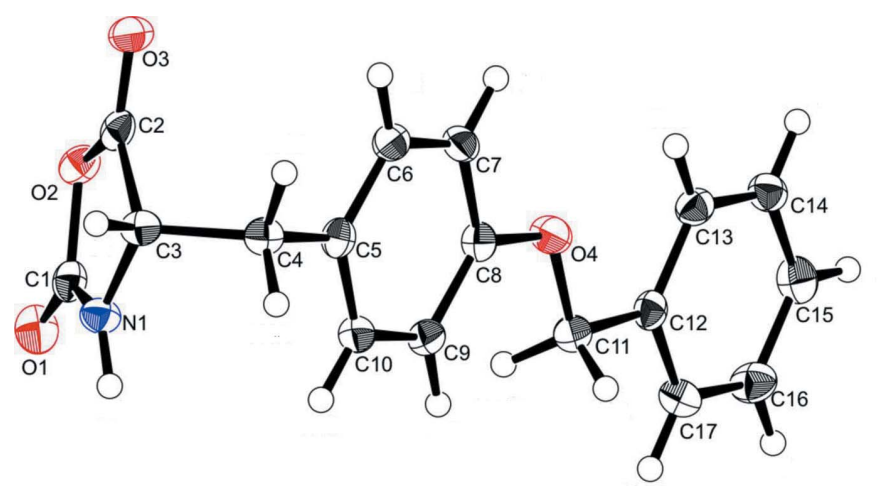

Figure 1

The molecular structure of the title compound, showing the atom labelling and $50 \%$ probability displacement ellipsoids.
Table 1

Hydrogen-bond geometry $\left(\AA,^{\circ}\right)$.

$\mathrm{Cg}$ is the centroid of the $\mathrm{C} 12-\mathrm{C} 17$ benzyloxy ring.

\begin{tabular}{lllll}
\hline$D-\mathrm{H} \cdots A$ & $D-\mathrm{H}$ & $\mathrm{H} \cdots A$ & $D \cdots A$ & $D-\mathrm{H} \cdots A$ \\
\hline $\mathrm{N} 1-\mathrm{H} 1 \cdots \mathrm{O} 3^{\mathrm{i}}$ & $0.88(3)$ & $2.09(3)$ & $2.885(2)$ & $150(2)$ \\
$\mathrm{C} 3-\mathrm{H} 3 \cdots 3^{\mathrm{ii}}$ & 1.00 & 2.50 & $3.410(3)$ & 151 \\
$\mathrm{C} 6-\mathrm{H} 6 \cdots \mathrm{Cg}^{\mathrm{iii}}$ & 0.95 & 2.89 & $3.546(3)$ & 127 \\
\hline
\end{tabular}

Symmetry codes: (i) $x, y+1, z$; (ii) $-x+1, y+\frac{1}{2},-z+2$; (iii) $-x+1, y-\frac{1}{2},-z+1$.

\section{Database survey}

A search of the Cambridge Structural Database (Version 5.37, update May 2016; Groom et al., 2016) revealed the presence of 15 hits for 4-methylene-oxazolidine-2,5-dione or 4-ethyl-4methylene-oxazolidine-2,5-dione derivatives. A number of these compounds involve amino acid side chains (amino acid NCAs). There are four compounds in which a benzyl group side chain is bonded to atom $\mathrm{C} 4$ in the oxazolidine-2,5-dione ring, viz $N$-carboxy-L-phenylalanine anhydride (KIXSUF; Kanazawa, 2000), $N$-carboxy-DL-phenylalanine anhydride (RESSUD; Kanazawa et al., 1997), $N$-carboxy-( $R$ )-phenylalanine anhydride 3-(2-thienyl) alanine- $N$-carboxyanhydride (SAPYEO; Nery et al.,2005) and $C^{\alpha}$-ethyl-( $(S)$-phenylalanine$N$-carboxyanhydride (ZATWEW; Crisma et al.,1995). In these compounds, the dihedral angles between oxazolidine-2,5dione ring mean plane and the benzene ring are very similar, viz $58.42(11)^{\circ}$ in the title compound, $59.34(15)^{\circ}$ in KIXSUF, $55.8(2)$ and $54.7(2)^{\circ}$ in RESSUD, $51.7(7), 50.6(7)^{\circ}$ in SAPYEO and $58.8(7)^{\circ}$ in ZATWEW. Intermolecular

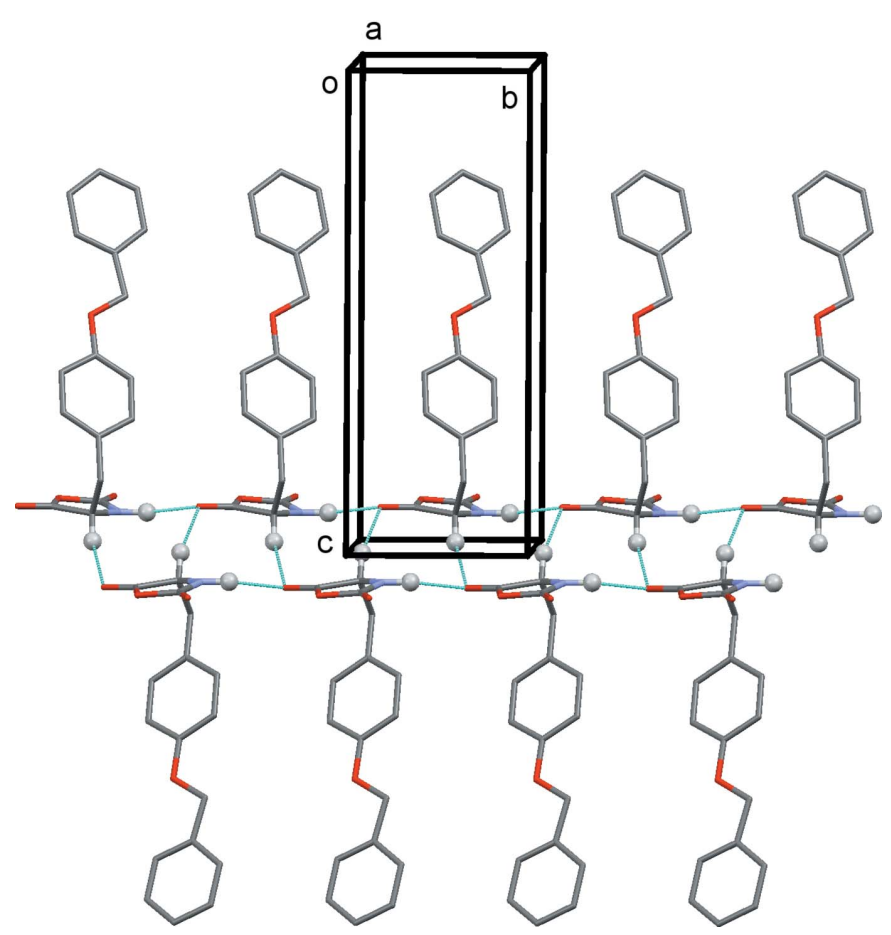

Figure 2

A partial view along the $a$ axis of the crystal packing of the title compound. The hydrogen bonds are shown as dashed lines (see Table 1). For clarity, only H atoms H1 and H3 (grey balls) have been included. 


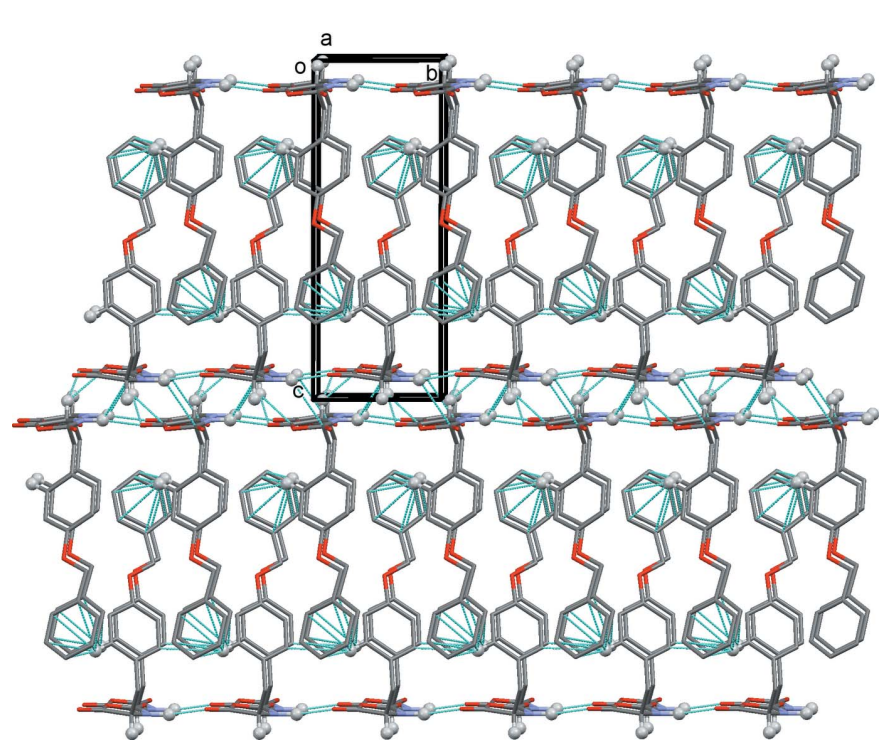

Figure 3

A view along the $a$ axis of the crystal packing of the title compound. The hydrogen bonds and $\mathrm{C}-\mathrm{H} \cdots \pi$ interactions are shown as dashed lines (see Table 1). For clarity, only H atoms H1 and H3 and H6 (grey balls) have been included.

hydrogen bonds are formed between the imino group and the carbonyl $\mathrm{O}$ atom in position 2 of the oxazolidine ring in the title compound and in ZATWEW. On the other hand, they are formed between the imino group and the carbonyl $\mathrm{O}$ atom at position 5 of the oxazolidine ring in KIXSUF and RESSUD.

\section{Synthesis and crystallization}

Reagent-grade $O$-benzyl-L-tyrosine (OBLT) (Product Code B3210; Tokyo Kasei Co. Ltd.) was used as received. The title compound was synthesized by the reaction of OBLT with triphosgene in tetrahydrofuran, as reported previously for the synthesis of BLA NCA (Kanazawa \& Magoshi, 2003). The reaction product was recrystallized slowly in a mixture of ethyl acetate and hexane $(1: 50 \mathrm{v} / \mathrm{v})$, avoiding moisture contamination, and gave colourless needle-shaped crystals.

\section{Refinement}

Crystal data, data collection and structure refinement details are summarized in Table 2. The $\mathrm{N}$-bound $\mathrm{H}$ atom was located in a difference-Fourier map and refined with a distance restraint of $\mathrm{N}-\mathrm{H}=0.88(4) \AA$, with $U_{\text {iso }}(\mathrm{H})=1.14 U_{\text {eq }}(\mathrm{N})$. Cbound $\mathrm{H}$ atoms were positioned geometrically and treated as riding: $\mathrm{C}-\mathrm{H}=0.95-1.00 \AA$ with $U_{\text {iso }}(\mathrm{H})=1.2 U_{\text {eq }}(\mathrm{C})$.

\section{Acknowledgements}

HK thanks the Rigaku Corporation, Tokyo, Japan, for assistance with the data collection of the title compound and $\mathrm{Dr}$
Table 2

Experimental details.

\begin{tabular}{|c|c|}
\hline \multicolumn{2}{|l|}{ Crystal data } \\
\hline Chemical formula & $\mathrm{C}_{17} \mathrm{H}_{15} \mathrm{NO}_{4}$ \\
\hline$M_{\mathrm{r}}$ & 297.30 \\
\hline Crystal system, space group & Monoclinic, $P 2_{1}$ \\
\hline Temperature $(\mathrm{K})$ & 123 \\
\hline$a, b, c(\AA)$ & $7.7388(5), 5.9128(4), 15.7769(10)$ \\
\hline$\beta\left(^{\circ}\right)$ & $96.390(2)$ \\
\hline$V\left(\AA^{3}\right)$ & $717.43(8)$ \\
\hline$Z$ & 2 \\
\hline Radiation type & Мо $K \alpha$ \\
\hline$\mu\left(\mathrm{mm}^{-1}\right)$ & 0.10 \\
\hline Crystal size $(\mathrm{mm})$ & $0.26 \times 0.13 \times 0.10$ \\
\hline \multicolumn{2}{|l|}{ Data collection } \\
\hline Diffractometer & Rigaku R-AXIS RAPID \\
\hline Absorption correction & $\begin{array}{l}\text { Multi-scan (ABSCOR; Higashi, } \\
\text { 1995) }\end{array}$ \\
\hline$T_{\min }, T_{\max }$ & $0.975,0.990$ \\
\hline $\begin{array}{l}\text { No. of measured, independent and } \\
\text { observed }[I>2 \sigma(I)] \text { reflections }\end{array}$ & $6593,1635,1444$ \\
\hline$R_{\text {int }}$ & 0.034 \\
\hline$(\sin \theta / \lambda)_{\max }\left(\AA^{-1}\right)$ & 0.628 \\
\hline \multicolumn{2}{|l|}{ Refinement } \\
\hline$R\left[F^{2}>2 \sigma\left(F^{2}\right)\right], w R\left(F^{2}\right), S$ & $0.031,0.068,1.10$ \\
\hline No. of reflections & 1635 \\
\hline No. of parameters & 202 \\
\hline No. of restraints & 1 \\
\hline $\mathrm{H}$-atom treatment & $\begin{array}{l}\mathrm{H} \text { atoms treated by a mixture of } \\
\text { independent and constrained } \\
\text { refinement }\end{array}$ \\
\hline$\Delta \rho_{\max }, \Delta \rho_{\min }\left(\mathrm{e} \AA^{-3}\right)$ & $0.20,-0.18$ \\
\hline
\end{tabular}

Computer programs: RAPID-AUTO (Rigaku, 2009), SHELXS97 and SHELXL97 (Sheldrick, 2008), CrystalStructure (Rigaku, 2009) and Mercury (Macrae et al., 2008).

Hidehiro Uekusa of Tokyo Institute of Technology for assistance with the checking of the crystal-structure analysis of the title compound.

\section{References}

Crisma, M., Valle, G., Formaggio, F., Toniolo, C. \& Kamphui, J. (1995). Z. Kristallogr. 210, 634-635.

Groom, C. R., Bruno, I. J., Lightfoot, M. P. \& Ward, S. C. (2016). Acta Cryst. B72, 171-179.

Higashi, T. (1995). ABSCOR. Rigaku Corporation, Tokyo, Japan.

Kanazawa, H. (2000). Acta Cryst. C56, 469-470.

Kanazawa, H. \& Inada, A. (2017). Acta Cryst. E73, 445-447.

Kanazawa, H. \& Magoshi, J. (2003). Acta Cryst. C59, o159-o161.

Kanazawa, H. \& Sato, Y. (1996). Science Reports, Fukushima University. 59, 13-17.

Kanazawa, H., Uekusa, H. \& Ohashi, Y. (1997). Acta Cryst. C53, 1154-1156.

Kricheldorf, H. R. (2006). Angew. Chem. Int. Ed. 45, 5752-5784.

Macrae, C. F., Bruno, I. J., Chisholm, J. A., Edgington, P. R., McCabe, P., Pidcock, E., Rodriguez-Monge, L., Taylor, R., van de Streek, J. \& Wood, P. A. (2008). J. Appl. Cryst. 41, 466-470.

Nery, J. G., Bolbach, G., Weissbuch, G. \& Lahav, M. (2005). Chem. Eur. J. 11, 3039-3048.

Rigaku (2009). RAPID-AUTO and CrystalStructure, Rigaku Corporation. Tokyo, Japan.

Sheldrick, G. M. (2008). Acta Cryst. A64, 112-122. 


\section{supporting information}

Acta Cryst. (2017). E73, 553-555 [https://doi.org/10.1107/S2056989017004236]

\section{Crystal structure of O-benzyl-L-tyrosine $\mathrm{N}$-carboxy anhydride}

\section{Aya Inada and Hitoshi Kanazawa}

\section{Computing details}

Data collection: RAPID-AUTO (Rigaku, 2009); cell refinement: RAPID-AUTO (Rigaku, 2009); data reduction: RAPIDAUTO (Rigaku, 2009); program(s) used to solve structure: SHELXS97 (Sheldrick, 2008); program(s) used to refine structure: SHELXL97 (Sheldrick, 2008); molecular graphics: CrystalStructure (Rigaku, 2009) and Mercury (Macrae et al., 2008); software used to prepare material for publication: CrystalStructure (Rigaku, 2009).

(S)-4-[4-(Benzyloxy)benzyl] oxazolidine-2,5-dione

Crystal data

$\mathrm{C}_{17} \mathrm{H}_{15} \mathrm{NO}_{4}$

$M_{r}=297.30$

Monoclinic, $P 2_{1}$

Hall symbol: P 2yb

$a=7.7388(5) \AA$

$b=5.9128(4) \AA$

$c=15.7769(10) \AA$

$\beta=96.390(2)^{\circ}$

$V=717.43(8) \AA^{3}$

$Z=2$

\section{Data collection}

Rigaku R-AXIS RAPID diffractometer

Radiation source: fine-focus sealed tube Graphite monochromator

Detector resolution: 10.0 pixels $\mathrm{mm}^{-1}$

$\omega$-scan

Absorption correction: multi-scan

(ABSCOR; Higashi, 1995)

$T_{\text {min }}=0.975, T_{\text {max }}=0.990$

Refinement

Refinement on $F^{2}$

Least-squares matrix: full

$R\left[F^{2}>2 \sigma\left(F^{2}\right)\right]=0.031$

$w R\left(F^{2}\right)=0.068$

$S=1.10$

1635 reflections

202 parameters

1 restraint

Primary atom site location: structure-invariant direct methods
$F(000)=312$

$D_{\mathrm{x}}=1.376 \mathrm{Mg} \mathrm{m}^{-3}$

Mo $K \alpha$ radiation, $\lambda=0.71075 \AA$

Cell parameters from 7077 reflections

$\theta=3.5-27.4^{\circ}$

$\mu=0.10 \mathrm{~mm}^{-1}$

$T=123 \mathrm{~K}$

Needle, colourless

$0.26 \times 0.13 \times 0.10 \mathrm{~mm}$

6593 measured reflections

1635 independent reflections

1444 reflections with $I>2 \sigma(I)$

$R_{\text {int }}=0.034$

$\theta_{\max }=26.5^{\circ}, \theta_{\min }=3.5^{\circ}$

$h=-9 \rightarrow 9$

$k=-7 \rightarrow 7$

$l=-19 \rightarrow 19$

Secondary atom site location: difference Fourier map

Hydrogen site location: inferred from neighbouring sites

$\mathrm{H}$ atoms treated by a mixture of independent and constrained refinement

$w=1 /\left[\sigma^{2}\left(F_{\mathrm{o}}^{2}\right)+(0.0302 P)^{2}+0.1082 P\right]$ where $P=\left(F_{\mathrm{o}}^{2}+2 F_{\mathrm{c}}^{2}\right) / 3$

$(\Delta / \sigma)_{\max }<0.001$

$\Delta \rho_{\max }=0.20 \mathrm{e} \AA^{-3}$

$\Delta \rho_{\min }=-0.18$ e $\AA^{-3}$ 


\section{Special details}

Geometry. All esds (except the esd in the dihedral angle between two 1.s. planes) are estimated using the full covariance matrix. The cell esds are taken into account individually in the estimation of esds in distances, angles and torsion angles; correlations between esds in cell parameters are only used when they are defined by crystal symmetry. An approximate (isotropic) treatment of cell esds is used for estimating esds involving l.s. planes.

Refinement. Refinement of $\mathrm{F}^{2}$ against ALL reflections. The weighted R-factor $\mathrm{wR}$ and goodness of fit $\mathrm{S}$ are based on $\mathrm{F}^{2}$, conventional $R$-factors $R$ are based on $F$, with $F$ set to zero for negative $F^{2}$. The threshold expression of $F^{2}>2 \operatorname{sigma}\left(\mathrm{F}^{2}\right)$ is used only for calculating R-factors(gt) etc. and is not relevant to the choice of reflections for refinement. R-factors based on $\mathrm{F}^{2}$ are statistically about twice as large as those based on F, and R- factors based on ALL data will be even larger.

Fractional atomic coordinates and isotropic or equivalent isotropic displacement parameters $\left(\hat{A}^{2}\right)$

\begin{tabular}{|c|c|c|c|c|}
\hline & $x$ & $y$ & $z$ & $U_{\text {iso }} * / U_{\text {eq }}$ \\
\hline $\mathrm{O} 1$ & $1.08406(18)$ & $0.6360(3)$ & $0.91435(9)$ & $0.0310(4)$ \\
\hline $\mathrm{O} 2$ & 0.89115 (18) & 0.3443 & $0.91282(8)$ & 0.0238 \\
\hline $\mathrm{O} 3$ & $0.6500(2)$ & 0.1393 & $0.92135(9)$ & $0.0290(4)$ \\
\hline $\mathrm{O} 4$ & $0.70846(18)$ & $0.5324(3)$ & $0.52871(9)$ & 0.0265 (4) \\
\hline N1 & $0.7966(2)$ & 0.6908 & $0.93804(11)$ & $0.0229(4)$ \\
\hline H1 & $0.792(3)$ & $0.840(6)$ & $0.9383(16)$ & $0.026^{*}$ \\
\hline $\mathrm{C} 1$ & $0.9379(3)$ & $0.5765(4)$ & $0.92122(12)$ & $0.0234(5)$ \\
\hline $\mathrm{C} 2$ & $0.7201(3)$ & $0.3211(4)$ & $0.92381(12)$ & $0.0221(5)$ \\
\hline $\mathrm{C} 3$ & $0.6426(3)$ & 0.5499 & $0.93569(13)$ & $0.0213(4)$ \\
\hline H3 & 0.5941 & 0.5568 & 0.9918 & $0.026^{*}$ \\
\hline $\mathrm{C} 4$ & $0.4993(2)$ & $0.6067(4)$ & $0.86274(12)$ & $0.0222(5)$ \\
\hline $\mathrm{H} 4 \mathrm{~A}$ & 0.4003 & 0.5022 & 0.8659 & $0.027 *$ \\
\hline H4B & 0.4570 & 0.7622 & 0.8712 & $0.027 *$ \\
\hline C5 & $0.5591(2)$ & 0.5901 (4) & $0.77459(12)$ & $0.0212(5)$ \\
\hline C6 & $0.5233(3)$ & $0.3969(4)$ & $0.72446(13)$ & $0.0227(5)$ \\
\hline H6 & 0.4620 & 0.2743 & 0.7461 & $0.027 *$ \\
\hline $\mathrm{C} 7$ & $0.5764(3)$ & $0.3826(4)$ & 0.64309 (13) & $0.0229(5)$ \\
\hline H7 & 0.5524 & 0.2500 & 0.6098 & $0.027 *$ \\
\hline $\mathrm{C} 8$ & $0.6648(2)$ & $0.5620(4)$ & $0.61046(13)$ & 0.0218 \\
\hline C9 & 0.7028 & 0.7545 & $0.65934(13)$ & $0.0230(5)$ \\
\hline H9 & 0.7644 & 0.8766 & 0.6376 & $0.028 *$ \\
\hline $\mathrm{C} 10$ & $0.6491(3)$ & $0.7661(4)$ & $0.74112(13)$ & $0.0233(5)$ \\
\hline H10 & 0.6750 & 0.8978 & 0.7746 & $0.028 *$ \\
\hline C11 & 0.7964 & $0.7139(4)$ & $0.49268(12)$ & $0.0236(5)$ \\
\hline H11A & 0.9058 & 0.7481 & 0.5292 & $0.028 *$ \\
\hline H11B & 0.7226 & 0.8510 & 0.4896 & $0.028 *$ \\
\hline C12 & $0.8365(2)$ & $0.6502(4)$ & $0.40454(13)$ & $0.0215(4)$ \\
\hline C13 & 0.7879 & $0.4450(4)$ & $0.36549(13)$ & $0.0231(5)$ \\
\hline H13 & 0.7276 & 0.3355 & 0.3950 & $0.028 *$ \\
\hline C14 & 0.8277 & $0.4004(4)$ & $0.28317(13)$ & $0.0245(5)$ \\
\hline H14 & 0.7950 & 0.2597 & 0.2571 & $0.029 *$ \\
\hline C15 & $0.9141(3)$ & $0.5582(4)$ & $0.23910(13)$ & $0.0281(5)$ \\
\hline H15 & 0.9394 & 0.5271 & 0.1827 & $0.034 *$ \\
\hline $\mathrm{C} 16$ & $0.9636(3)$ & $0.7619(4)$ & $0.27748(14)$ & $0.0293(5)$ \\
\hline H16 & 1.0239 & 0.8706 & 0.2476 & $0.035^{*}$ \\
\hline
\end{tabular}




$\begin{array}{lllll}\mathrm{C} 17 & 0.9253(3) & 0.8080(4) & 0.35980(13) & 0.0265(5) \\ \mathrm{H} 17 & 0.9598 & 0.9482 & 0.3858 & 0.032^{*}\end{array}$

Atomic displacement parameters $\left(\AA^{2}\right)$

\begin{tabular}{lllllll}
\hline & $U^{11}$ & $U^{22}$ & $U^{33}$ & $U^{12}$ & $U^{13}$ & $U^{23}$ \\
\hline O1 & $0.0254(8)$ & $0.0395(10)$ & $0.0284(7)$ & $-0.0028(8)$ & $0.0048(6)$ & $0.0012(7)$ \\
O2 & $0.0264(7)$ & $0.0216(8)$ & $0.0242(7)$ & $0.0054(7)$ & $0.0067(6)$ & $0.0018(7)$ \\
O3 & $0.0374(9)$ & $0.0201(8)$ & $0.0295(8)$ & $-0.0022(7)$ & $0.0040(7)$ & $0.0011(7)$ \\
O4 & $0.0333(8)$ & $0.0254(9)$ & $0.0214(7)$ & $-0.0067(7)$ & $0.0060(6)$ & $-0.0013(6)$ \\
N1 & $0.0234(9)$ & $0.0190(10)$ & $0.0264(8)$ & $0.0002(8)$ & $0.0030(7)$ & $-0.0026(8)$ \\
C1 & $0.0263(11)$ & $0.0272(12)$ & $0.0164(9)$ & $0.0002(10)$ & $0.0020(8)$ & $0.0030(9)$ \\
C2 & $0.0287(11)$ & $0.0244(12)$ & $0.0138(9)$ & $0.0034(10)$ & $0.0051(8)$ & $0.0031(8)$ \\
C3 & $0.0249(10)$ & $0.0194(11)$ & $0.0208(9)$ & $-0.0005(9)$ & $0.0084(8)$ & $-0.0008(9)$ \\
C4 & $0.0198(9)$ & $0.0218(12)$ & $0.0254(10)$ & $0.0014(9)$ & $0.0044(8)$ & $-0.0006(9)$ \\
C5 & $0.0156(9)$ & $0.0241(13)$ & $0.0232(9)$ & $0.0043(9)$ & $-0.0002(8)$ & $0.0016(9)$ \\
C6 & $0.0189(10)$ & $0.0225(12)$ & $0.0264(10)$ & $-0.0013(9)$ & $0.0008(8)$ & $0.0031(9)$ \\
C7 & $0.0209(10)$ & $0.0209(12)$ & $0.0258(10)$ & $-0.0011(9)$ & $-0.0024(8)$ & $-0.0025(9)$ \\
C8 & $0.0204(10)$ & $0.0243(11)$ & $0.0203(9)$ & $0.0012(10)$ & $0.0012(8)$ & $0.0001(9)$ \\
C9 & $0.0243(10)$ & $0.0212(11)$ & $0.0232(10)$ & $-0.0020(9)$ & $0.0012(8)$ & $0.0033(9)$ \\
C10 & $0.0243(11)$ & $0.0207(12)$ & $0.0242(10)$ & $0.0004(9)$ & $-0.0009(8)$ & $-0.0004(9)$ \\
C11 & $0.0250(10)$ & $0.0223(12)$ & $0.0234(10)$ & $-0.0022(9)$ & $0.0020(8)$ & $0.0017(9)$ \\
C12 & $0.0172(9)$ & $0.0249(11)$ & $0.0217(9)$ & $0.0013(9)$ & $-0.0006(8)$ & $0.0028(9)$ \\
C13 & $0.0212(11)$ & $0.0216(11)$ & $0.0265(10)$ & $0.0009(9)$ & $0.0021(8)$ & $0.0028(9)$ \\
C14 & $0.0223(11)$ & $0.0228(12)$ & $0.0277(10)$ & $0.0006(9)$ & $0.0001(9)$ & $-0.0018(9)$ \\
C15 & $0.0263(11)$ & $0.0344(13)$ & $0.0241(10)$ & $0.0029(11)$ & $0.0045(8)$ & $-0.0038(10)$ \\
C16 & $0.0332(12)$ & $0.0275(13)$ & $0.0287(11)$ & $-0.0040(10)$ & $0.0105(9)$ & $0.0038(10)$ \\
C17 & $0.0264(11)$ & $0.0240(13)$ & $0.0290(11)$ & $-0.0039(10)$ & $0.0024(9)$ & $-0.0024(10)$ \\
& & & & & &
\end{tabular}

Geometric parameters $\left(\AA,{ }^{\circ}\right)$

\begin{tabular}{llll}
\hline $\mathrm{O} 1-\mathrm{C} 1$ & $1.201(2)$ & $\mathrm{C} 7-\mathrm{H} 7$ & 0.9500 \\
$\mathrm{O} 2-\mathrm{C} 2$ & $1.361(2)$ & $\mathrm{C} 8-\mathrm{C} 9$ & $1.388(3)$ \\
$\mathrm{O} 2-\mathrm{C} 1$ & $1.422(3)$ & $\mathrm{C} 9-\mathrm{C} 10$ & $1.400(3)$ \\
$\mathrm{O} 3-\mathrm{C} 2$ & $1.202(3)$ & $\mathrm{C} 9-\mathrm{H} 9$ & 0.9500 \\
$\mathrm{O} 4-\mathrm{C} 8$ & $1.380(2)$ & $\mathrm{C} 10-\mathrm{H} 10$ & 0.9500 \\
$\mathrm{O} 4-\mathrm{C} 11$ & $1.423(3)$ & $\mathrm{C} 11-\mathrm{C} 12$ & $1.506(3)$ \\
$\mathrm{N} 1-\mathrm{C} 1$ & $1.337(3)$ & $\mathrm{C} 11-\mathrm{H} 11 \mathrm{~A}$ & 0.9900 \\
$\mathrm{~N} 1-\mathrm{C} 3$ & $1.451(3)$ & $\mathrm{C} 11-\mathrm{H} 11 \mathrm{~B}$ & 0.9900 \\
$\mathrm{~N} 1-\mathrm{H} 1$ & $0.88(3)$ & $\mathrm{C} 12-\mathrm{C} 13$ & $1.393(3)$ \\
$\mathrm{C} 2-\mathrm{C} 3$ & $1.500(3)$ & $\mathrm{C} 12-\mathrm{C} 17$ & $1.396(3)$ \\
$\mathrm{C} 3-\mathrm{C} 4$ & $1.544(3)$ & $\mathrm{C} 13-\mathrm{C} 14$ & $1.393(3)$ \\
$\mathrm{C} 3-\mathrm{H} 3$ & 1.0000 & $\mathrm{C} 13-\mathrm{H} 13$ & 0.9500 \\
$\mathrm{C} 4-\mathrm{C} 5$ & $1.517(3)$ & $\mathrm{C} 14-\mathrm{C} 15$ & $1.380(3)$ \\
$\mathrm{C} 4-\mathrm{H} 4 \mathrm{~A}$ & 0.9900 & $\mathrm{C} 14-\mathrm{H} 14$ & 0.9500 \\
$\mathrm{C} 4-\mathrm{H} 4 \mathrm{~B}$ & 0.9900 & $\mathrm{C} 15-\mathrm{C} 16$ & $1.383(3)$ \\
$\mathrm{C} 5-\mathrm{C} 10$ & $1.389(3)$ & $\mathrm{C} 15-\mathrm{H} 15$ & 0.9500 \\
$\mathrm{C} 5-\mathrm{C} 6$ & $1.399(3)$ & $\mathrm{C} 16-\mathrm{C} 17$ & $1.391(3)$
\end{tabular}




\begin{tabular}{|c|c|c|c|}
\hline $\mathrm{C} 6-\mathrm{C} 7$ & $1.394(3)$ & $\mathrm{C} 16-\mathrm{H} 16$ & 0.9500 \\
\hline $\mathrm{C} 6-\mathrm{H} 6$ & 0.9500 & C17-H17 & 0.9500 \\
\hline $\mathrm{C} 7-\mathrm{C} 8$ & $1.391(3)$ & & \\
\hline $\mathrm{C} 2-\mathrm{O} 2-\mathrm{C} 1$ & $109.05(16)$ & $\mathrm{O} 4-\mathrm{C} 8-\mathrm{C} 7$ & $115.5(2)$ \\
\hline $\mathrm{C} 8-\mathrm{O} 4-\mathrm{C} 11$ & $117.10(17)$ & $\mathrm{C} 9-\mathrm{C} 8-\mathrm{C} 7$ & $120.15(18)$ \\
\hline $\mathrm{C} 1-\mathrm{N} 1-\mathrm{C} 3$ & $113.10(19)$ & $\mathrm{C} 8-\mathrm{C} 9-\mathrm{C} 10$ & $119.05(19)$ \\
\hline $\mathrm{C} 1-\mathrm{N} 1-\mathrm{H} 1$ & $122.7(16)$ & $\mathrm{C} 8-\mathrm{C} 9-\mathrm{H} 9$ & 120.5 \\
\hline $\mathrm{C} 3-\mathrm{N} 1-\mathrm{H} 1$ & $122.7(16)$ & $\mathrm{C} 10-\mathrm{C} 9-\mathrm{H} 9$ & 120.5 \\
\hline $\mathrm{O} 1-\mathrm{C} 1-\mathrm{N} 1$ & $132.0(2)$ & $\mathrm{C} 5-\mathrm{C} 10-\mathrm{C} 9$ & $121.8(2)$ \\
\hline $\mathrm{O} 1-\mathrm{C} 1-\mathrm{O} 2$ & $120.44(19)$ & $\mathrm{C} 5-\mathrm{C} 10-\mathrm{H} 10$ & 119.1 \\
\hline $\mathrm{N} 1-\mathrm{C} 1-\mathrm{O} 2$ & $107.53(17)$ & $\mathrm{C} 9-\mathrm{C} 10-\mathrm{H} 10$ & 119.1 \\
\hline $\mathrm{O} 3-\mathrm{C} 2-\mathrm{O} 2$ & $121.8(2)$ & $\mathrm{O} 4-\mathrm{C} 11-\mathrm{C} 12$ & $109.70(18)$ \\
\hline $\mathrm{O} 3-\mathrm{C} 2-\mathrm{C} 3$ & $128.69(18)$ & $\mathrm{O} 4-\mathrm{C} 11-\mathrm{H} 11 \mathrm{~A}$ & 109.7 \\
\hline $\mathrm{O} 2-\mathrm{C} 2-\mathrm{C} 3$ & $109.44(18)$ & $\mathrm{C} 12-\mathrm{C} 11-\mathrm{H} 11 \mathrm{~A}$ & 109.7 \\
\hline $\mathrm{N} 1-\mathrm{C} 3-\mathrm{C} 2$ & $100.53(16)$ & $\mathrm{O} 4-\mathrm{C} 11-\mathrm{H} 11 \mathrm{~B}$ & 109.7 \\
\hline $\mathrm{N} 1-\mathrm{C} 3-\mathrm{C} 4$ & $114.45(17)$ & $\mathrm{C} 12-\mathrm{C} 11-\mathrm{H} 11 \mathrm{~B}$ & 109.7 \\
\hline $\mathrm{C} 2-\mathrm{C} 3-\mathrm{C} 4$ & $111.52(18)$ & $\mathrm{H} 11 \mathrm{~A}-\mathrm{C} 11-\mathrm{H} 11 \mathrm{~B}$ & 108.2 \\
\hline $\mathrm{N} 1-\mathrm{C} 3-\mathrm{H} 3$ & 110.0 & $\mathrm{C} 13-\mathrm{C} 12-\mathrm{C} 17$ & $118.84(18)$ \\
\hline $\mathrm{C} 2-\mathrm{C} 3-\mathrm{H} 3$ & 110.0 & $\mathrm{C} 13-\mathrm{C} 12-\mathrm{C} 11$ & $123.57(19)$ \\
\hline $\mathrm{C} 4-\mathrm{C} 3-\mathrm{H} 3$ & 110.0 & $\mathrm{C} 17-\mathrm{C} 12-\mathrm{C} 11$ & $117.6(2)$ \\
\hline $\mathrm{C} 5-\mathrm{C} 4-\mathrm{C} 3$ & $113.72(15)$ & $\mathrm{C} 14-\mathrm{C} 13-\mathrm{C} 12$ & $120.03(19)$ \\
\hline $\mathrm{C} 5-\mathrm{C} 4-\mathrm{H} 4 \mathrm{~A}$ & 108.8 & $\mathrm{C} 14-\mathrm{C} 13-\mathrm{H} 13$ & 120.0 \\
\hline $\mathrm{C} 3-\mathrm{C} 4-\mathrm{H} 4 \mathrm{~A}$ & 108.8 & $\mathrm{C} 12-\mathrm{C} 13-\mathrm{H} 13$ & 120.0 \\
\hline $\mathrm{C} 5-\mathrm{C} 4-\mathrm{H} 4 \mathrm{~B}$ & 108.8 & $\mathrm{C} 15-\mathrm{C} 14-\mathrm{C} 13$ & $120.8(2)$ \\
\hline $\mathrm{C} 3-\mathrm{C} 4-\mathrm{H} 4 \mathrm{~B}$ & 108.8 & $\mathrm{C} 15-\mathrm{C} 14-\mathrm{H} 14$ & 119.6 \\
\hline $\mathrm{H} 4 \mathrm{~A}-\mathrm{C} 4-\mathrm{H} 4 \mathrm{~B}$ & 107.7 & $\mathrm{C} 13-\mathrm{C} 14-\mathrm{H} 14$ & 119.6 \\
\hline $\mathrm{C} 10-\mathrm{C} 5-\mathrm{C} 6$ & $118.23(17)$ & $\mathrm{C} 14-\mathrm{C} 15-\mathrm{C} 16$ & $119.63(19)$ \\
\hline $\mathrm{C} 10-\mathrm{C} 5-\mathrm{C} 4$ & $121.1(2)$ & $\mathrm{C} 14-\mathrm{C} 15-\mathrm{H} 15$ & 120.2 \\
\hline $\mathrm{C} 6-\mathrm{C} 5-\mathrm{C} 4$ & $120.62(18)$ & $\mathrm{C} 16-\mathrm{C} 15-\mathrm{H} 15$ & 120.2 \\
\hline $\mathrm{C} 7-\mathrm{C} 6-\mathrm{C} 5$ & $120.66(19)$ & $\mathrm{C} 15-\mathrm{C} 16-\mathrm{C} 17$ & $120.2(2)$ \\
\hline $\mathrm{C} 7-\mathrm{C} 6-\mathrm{H} 6$ & 119.7 & $\mathrm{C} 15-\mathrm{C} 16-\mathrm{H} 16$ & 119.9 \\
\hline $\mathrm{C} 5-\mathrm{C} 6-\mathrm{H} 6$ & 119.7 & $\mathrm{C} 17-\mathrm{C} 16-\mathrm{H} 16$ & 119.9 \\
\hline $\mathrm{C} 8-\mathrm{C} 7-\mathrm{C} 6$ & $120.1(2)$ & $\mathrm{C} 16-\mathrm{C} 17-\mathrm{C} 12$ & $120.6(2)$ \\
\hline $\mathrm{C} 8-\mathrm{C} 7-\mathrm{H} 7$ & 119.9 & $\mathrm{C} 16-\mathrm{C} 17-\mathrm{H} 17$ & 119.7 \\
\hline $\mathrm{C} 6-\mathrm{C} 7-\mathrm{H} 7$ & 119.9 & $\mathrm{C} 12-\mathrm{C} 17-\mathrm{H} 17$ & 119.7 \\
\hline $\mathrm{O} 4-\mathrm{C} 8-\mathrm{C} 9$ & $124.34(19)$ & & \\
\hline $\mathrm{C} 3-\mathrm{N} 1-\mathrm{C} 1-\mathrm{O} 1$ & $176.8(2)$ & $\mathrm{C} 11-\mathrm{O} 4-\mathrm{C} 8-\mathrm{C} 7$ & $179.01(18)$ \\
\hline $\mathrm{C} 3-\mathrm{N} 1-\mathrm{C} 1-\mathrm{O} 2$ & $-4.5(2)$ & $\mathrm{C} 6-\mathrm{C} 7-\mathrm{C} 8-\mathrm{O} 4$ & $-178.74(18)$ \\
\hline $\mathrm{C} 2-\mathrm{O} 2-\mathrm{C} 1-\mathrm{O} 1$ & $179.56(17)$ & $\mathrm{C} 6-\mathrm{C} 7-\mathrm{C} 8-\mathrm{C} 9$ & $1.1(3)$ \\
\hline $\mathrm{C} 2-\mathrm{O} 2-\mathrm{C} 1-\mathrm{N} 1$ & $0.7(2)$ & $\mathrm{O} 4-\mathrm{C} 8-\mathrm{C} 9-\mathrm{C} 10$ & $178.99(19)$ \\
\hline $\mathrm{C} 1-\mathrm{O} 2-\mathrm{C} 2-\mathrm{O} 3$ & $-178.53(18)$ & $\mathrm{C} 7-\mathrm{C} 8-\mathrm{C} 9-\mathrm{C} 10$ & $-0.8(3)$ \\
\hline $\mathrm{C} 1-\mathrm{O} 2-\mathrm{C} 2-\mathrm{C} 3$ & $3.2(2)$ & $\mathrm{C} 6-\mathrm{C} 5-\mathrm{C} 10-\mathrm{C} 9$ & $0.4(3)$ \\
\hline $\mathrm{C} 1-\mathrm{N} 1-\mathrm{C} 3-\mathrm{C} 2$ & $6.0(2)$ & $\mathrm{C} 4-\mathrm{C} 5-\mathrm{C} 10-\mathrm{C} 9$ & $-179.00(19)$ \\
\hline $\mathrm{C} 1-\mathrm{N} 1-\mathrm{C} 3-\mathrm{C} 4$ & $-113.59(19)$ & $\mathrm{C} 8-\mathrm{C} 9-\mathrm{C} 10-\mathrm{C} 5$ & $0.1(3)$ \\
\hline $\mathrm{O} 3-\mathrm{C} 2-\mathrm{C} 3-\mathrm{N} 1$ & $176.5(2)$ & $\mathrm{C} 8-\mathrm{O} 4-\mathrm{C} 11-\mathrm{C} 12$ & $178.88(17)$ \\
\hline $\mathrm{O} 2-\mathrm{C} 2-\mathrm{C} 3-\mathrm{N} 1$ & $-5.4(2)$ & $\mathrm{O} 4-\mathrm{C} 11-\mathrm{C} 12-\mathrm{C} 13$ & $1.7(3)$ \\
\hline
\end{tabular}




\begin{tabular}{llll}
$\mathrm{O} 3-\mathrm{C} 2-\mathrm{C} 3-\mathrm{C} 4$ & $-61.8(3)$ & $\mathrm{O} 4-\mathrm{C} 11-\mathrm{C} 12-\mathrm{C} 17$ & $-179.17(18)$ \\
$\mathrm{O} 2-\mathrm{C} 2-\mathrm{C} 3-\mathrm{C} 4$ & $116.34(17)$ & $\mathrm{C} 17-\mathrm{C} 12-\mathrm{C} 13-\mathrm{C} 14$ & $-0.1(3)$ \\
$\mathrm{N} 1-\mathrm{C} 3-\mathrm{C} 4-\mathrm{C} 5$ & $58.2(2)$ & $\mathrm{C} 11-\mathrm{C} 12-\mathrm{C} 13-\mathrm{C} 14$ & $179.01(19)$ \\
$\mathrm{C} 2-\mathrm{C} 3-\mathrm{C} 4-\mathrm{C} 5$ & $-55.1(2)$ & $\mathrm{C} 12-\mathrm{C} 13-\mathrm{C} 14-\mathrm{C} 15$ & $-0.5(3)$ \\
$\mathrm{C} 3-\mathrm{C} 4-\mathrm{C} 5-\mathrm{C} 10$ & $-81.9(2)$ & $\mathrm{C} 13-\mathrm{C} 14-\mathrm{C} 15-\mathrm{C} 16$ & $0.8(3)$ \\
$\mathrm{C} 3-\mathrm{C} 4-\mathrm{C} 5-\mathrm{C} 6$ & $98.8(2)$ & $\mathrm{C} 14-\mathrm{C} 15-\mathrm{C} 16-\mathrm{C} 17$ & $-0.5(3)$ \\
$\mathrm{C} 10-\mathrm{C} 5-\mathrm{C} 6-\mathrm{C} 7$ & $-0.1(3)$ & $\mathrm{C} 15-\mathrm{C} 16-\mathrm{C} 17-\mathrm{C} 12$ & $-0.1(3)$ \\
$\mathrm{C} 4-\mathrm{C} 5-\mathrm{C} 6-\mathrm{C} 7$ & $179.27(18)$ & $\mathrm{C} 13-\mathrm{C} 12-\mathrm{C} 17-\mathrm{C} 16$ & $0.4(3)$ \\
$\mathrm{C} 5-\mathrm{C} 6-\mathrm{C} 7-\mathrm{C} 8$ & $-0.6(3)$ & $\mathrm{C} 11-\mathrm{C} 12-\mathrm{C} 17-\mathrm{C} 16$ & $-178.8(2)$ \\
$\mathrm{C} 11-\mathrm{O} 4-\mathrm{C} 8-\mathrm{C} 9$ & $-0.8(3)$ & & \\
\hline
\end{tabular}

Hydrogen-bond geometry $\left(\AA,{ }^{\circ}\right)$

$\mathrm{Cg}$ is the centroid of the $\mathrm{C} 12-\mathrm{C} 17$ benzyloxy ring.

\begin{tabular}{lllll}
\hline$D-\mathrm{H} \cdots A$ & $D-\mathrm{H}$ & $\mathrm{H} \cdots A$ & $D \cdots A$ & $D-\mathrm{H}^{\cdots} A A$ \\
\hline $\mathrm{N} 1-\mathrm{H} 1 \cdots \mathrm{O} 3^{\mathrm{i}}$ & $0.88(3)$ & $2.09(3)$ & $2.885(2)$ & $150(2)$ \\
$\mathrm{C} 3-\mathrm{H} 3 \cdots 3^{\mathrm{ii}}$ & 1.00 & 2.50 & $3.410(3)$ & 151 \\
$\mathrm{C} 6-\mathrm{H} 6 \cdots \mathrm{Cg}^{\mathrm{iii}}$ & 0.95 & 2.89 & $3.546(3)$ & 127 \\
\hline
\end{tabular}

Symmetry codes: (i) $x, y+1, z$; (ii) $-x+1, y+1 / 2,-z+2$; (iii) $-x+1, y-1 / 2,-z+1$. 